\title{
Development and Validation of a Novel Immune- Related Prognostic Model and the Potential Mechanism in Metastatic Synovial Sarcoma
}

\section{Yufeng Huang}

Sun Yat-sen University Cancer Center https://orcid.org/0000-0001-8543-901X

Ming Gong

Sun Yat-sen University Cancer Center

Qinglian Tang

Sun Yat-sen University Cancer Center

Hongmin Chen

Sun Yat-sen University Cancer Center

Jinxin $\mathrm{Hu}$

Sun Yat-sen University Cancer Center

\section{Chuangzhong Deng}

Sun Yat-sen University Cancer Center

Jiaming Lin

Sun Yat-sen University Cancer Center

Anfei Huang

Sun Yat-sen University Cancer Center

Yanyang $\mathrm{Xu}$

Sun Yat-sen University Cancer Center Huixiong Feng

Sun Yat-sen University Cancer Center

\section{Guohui Song}

Sun Yat-sen University Cancer Center

Huaiyuan Xu

Sun Yat-sen University Cancer Center

Jinchang Lu

Sun Yat-sen University Cancer Center

Xiaojun Zhu

Sun Yat-sen University Cancer Center

Jin Wang ( $\nabla$ wangjinbs@sysucc.org.cn )

Sun Yat-sen University Cancer Center 


\section{Research article}

Keywords: synovial sarcoma, immune-related genes, immune checkpoints, prognosis, tumour microenvironment

Posted Date: November 10th, 2021

DOI: https://doi.org/10.21203/rs.3.rs-1044503/v1

License: (c) (i) This work is licensed under a Creative Commons Attribution 4.0 International License.

Read Full License 


\section{Abstract}

Background: Several clinical trials have shown that immunotherapy plays a pivotal role in the treatment of patients with metastatic synovial sarcoma. Immune-related genes (IRGs) have been demonstrated to play an important role in tumorigenesis and tumour microenvironment formation. However, the clinical significance of IRGs in patients with synovial sarcoma(SS) is still unclear, and systematic analysis is lacking.

Methods: We downloaded the GSE40021 dataset from the GEO database, which included 30 cases of nonmetastatic and 28 cases of metastatic SS. Firstly,We combined the immune-related ImmPort gene set to search for SS related to metastatic and differentially expressed immune-related genes (DEIRGs). We then performed univariate Cox regression analysis from soft tissue sarcoma database in TCGA to identify DEIRGs that related to overall survival, and constructed an immune-related prognostic assessment model. We used the assessment model to evaluate the infiltration of immune cells in the tumour microenvironment through the sSGSEA algorithm. Finally, we collected tumour tissues in our centre to verify the RNA expression levels by real-time quantitative reverse transcription(RT-qPCR) analysis.

Results: The study screened a total of six DEIRGs which were closely related to prognosisin in metastatic SS. We then constructed an immune-related prognostic assessment model which was an independent prognostic factor different from other clinical features. Further analysis showed that there was no significant difference in the expression of several immune checkpoints between the two groups in the GSE40021 data. Moreover, the GREM2 and CTSS genes were significantly expressed in metastatic patients. Further verification of clinical SS tissues from our centre by RT-qPCR analysis demonstrated reduced infiltration of activated NK cells and macrophages but increased M2-type macrophages in metastatic patients.

Conclusion: The study successfully constructed an immune-related prognostic assessment model and probably explain the poor efficacy PD-1 inhibitors for SS patients. Together,the research deepens our understanding of the tumor immune microenvironment and proposed a new immune mechanism of metastatic SS.Advance intervention and reversal of microenvironmental changes are expected to delay metastasis and improve survival.

\section{Background}

Synovial sarcoma is a rare and aggressive malignant tumour derived from spindle-shaped mesenchymal tissue. The specific origin of this tumour tissue type has not been confirmed, but current research suggests that it may be derived from myoblasts and nerve or primitive mesenchymal cells[1]. Genetically, synovial sarcoma has a specific $t(X ; 18)(p 11.2 ; q 11.2)$ gene translocation and produces the SS18-SSX fusion gene. Synovial sarcoma accounts for $5-10 \%$ of soft tissue sarcomas, and the age of onset is mainly 15-40 years old[2, 3]. Synovial sarcoma is the most common soft tissue sarcoma in young people, except rhabdomyosarcoma. The current treatment of synovial sarcoma involves neoadjuvant 
chemotherapy combined with extensive surgical resection, and adjuvant chemotherapy or radiotherapy is also utilized according to the tumour stage and prognostic factors[4].

Synovial sarcoma and other soft tissue sarcomas have different biological behaviours, prognoses,and treatments.For example, synovial sarcoma is more sensitive to chemotherapy, and the molecular and immunotherapy targets are different. The lung is the most common site of initial recurrence after treatment.Once metastasis occurs, the median survival time is approximately 1 year[5].For patients with metastases who are resistant to advanced chemotherapy, immunotherapy may be one of the possible effective treatments. In the SARC028 clinical trial, patients with metastatic or surgically unresectable locally advanced sarcoma were treated with pembrolizumab $(200 \mathrm{mg})$ intravenously every three weeks, and the main endpoint of the study was the objective response rate of the tumour to the treatment. The results showed that undifferentiated sarcoma (UPS), myxoid liposarcoma(LPS), and synovial sarcoma(SS) had ORRs of $40 \%, 20 \%$, and $10 \%$,respectively[6]. In the clinical trial of ALLIANCE A091401,the patients with advanced soft tissue sarcoma were included in the same group to evaluate the objective response rate of nivolumab alone or in combination with ipilimumab. Among the effective types of sarcoma, the single-agent treatment group included Alveolar soft part sarcoma(ASPS) and Leiomyosarcoma(LMS), and the combined treatment group included undifferentiated sarcoma(UPS), Leiomyosarcoma(LMS), myxofibrosarcoma(MFS), and Anigosarcoma(AS)[7]. However, two subsequent clinical trials with expanded samples did not include SS. In adoptive cellular immunotherapy, up to $80 \%$ of patients with SS express NY-ESO-1 antigen, while the expression level is low in other normal mesenchymal cells. D'Angelo and colleagues genetically engineered autologous $T$ cell receptors to express NY-ESO-1 antibody to enhance the recognition and killing of target antigens. This clinical trial showed good safety and effectiveness, of which $50 \%(6 / 12)$ of patients showed an antitumour response[8]. Immunotherapy plays a pivotal role in patients with advanced synovial sarcoma, but screening these patients with effective immunotherapy has become a key issue in clinical treatment decision-making. At present, there is no definite biomarker or model that is effective in predicting the prognosis of advanced SS.

Based on bioinformatics analysis, the study aimed to screen the key metastatic genes associated with immune-related SS and to construct an immune-related prognostic model. First, we used the GSE40021 dataset combined with the ImmPort gene set to screen the DEIRGs of metastatic SS and performed functional enrichment analysis. We then used the soft tissue sarcoma database in TCGA to further screen the genes that were significantly related to prognosis to construct a risk assessment model. We also utilized the sSGSEA algorithm to compare immune cell infiltration in the tumour microenvironment between patients with high-risk scores and metastasis. We found that the infiltration of immune cell subpopulations overlapped significantly between the two groups. Among them, eosinophils, CD4+T lymphocytes, NK cells, and macrophages were significantly infiltrated in SS with poor prognosis, while the expression of most immune checkpoints were not significantly different.Finally,we found that the GREM2 and CTSS expressions(the other four genes were not significantly different) were significantly increased in metastatic SS from our centre. In addition, the infiltration of activated NK cells was reduced, but M2 macrophages were increased in these patients. 
The study successfully constructed an immune-related prognostic assessment model for metastatic SS that can predict the prognosis of patients and screen patients who may benefit from immunotherapy. By analysing the infiltration of immune cells in the tumour microenvironment, we found that the immune metastatic mechanism may be related to the polarization of M2 macrophages and reshaping of the immune microenvironment, resulting in a decrease in NK cell infiltration but not the escape of immune checkpoints. The model provides important anti-metastatic therapeutics for patients with advanced SS, which may reduce medical costs and delay the metastasis to distant organs, thereby improving the overall survival of patients. Thus, the model has important significance for clinical treatment decisions.

\section{Materials And Methods}

\section{Patients and datasets}

Clinical data: 1) Patients with synovial sarcoma who were admitted to the Department of Bone and Soft Tissue in our hospital from May 2017 to May 2018 and had at least three years of follow-up data were enrolled. According to statistics, there were 11 nonmetastatic cases and 5 metastatic cases. The general clinical data of the patients are shown in TABLE 1.2) Clinical data of high-level soft tissue sarcoma patients were downloaded from TCGA database. General clinical information was obtained for 263 patients (TABLE 2).

RNA-seq data: 1) The expression profile data for the GSE40021 dataset were obtained from the GEO database (https:www.ncbi.nlm.nih.gov/geo/), which is based on the GPL6480 platform (Agilent-014850 Whole Human Genome Microarray 4× 44K G4112F), including 28 cases of synovial sarcoma tissue samples that had metastasized at the time of consultation and 30 cases of non-metastatic samples.

\section{Identification of differentially expressed immune-related genes (DEIRGs) and enrichment analysis}

To screen for metastatic genes associated with immune-related synovial sarcoma, we used the GEO2R online web tool, which allows users to compare the expression data of different genes between metastatic and non-metastatic samples. We used the Wilcoxon test method and set the corrected $P$ value $<0.05$ and $\mid \log 2$-fold change $\mid \geq 1.0$ as significant for the DEGs. Among them, $D E G s$ with $\log F C>0$ were considered to be upregulated, while $\mathrm{DEG}$ s with $\log \mathrm{FC}<0$ were considered to be downregulated.We also download 1811 immune-related genes via the Immunology Database and Analysis Portal (ImmPort,https://www.immport.org/shared/genelists) database, which contains 17 immune categories based on various molecular function[9].Finally,we took the intersection between DEGs and Immunerelated gene sets (IRGs) in ImmPort gene sets to obtain DEIRGs. Heatmaps were generated using pheatmap package and volcano plots were also conducted in $\mathrm{R}$ software.

To evaluate the potential biologic functions of DEIRGs, Gene Ontology (GO) [10]and Kyoto Encyclopedia of Genes and Genomes (KEGG) pathway enrichment analysis[11] were performed by the cluster Profiler package in R. Functional categories with a adjusted $P$ value $<0.05$ were considered as significant 
pathways.GO function enrichment included biological process (BP), cell component (CC), and molecular function (MF). The top 10 functional enrichment results and signalling pathways were selected.

The DEIRGs list was used for protein-protein interaction (PPI) analysis in Cytoscape (v3.7.1, National Resource for Network Biology, https://cytoscape.org/) with default parameters[12]. Only individual networks with more than 10 nodes were included for Molecular COmplex DEtection (MCODE).

\section{Survival analysis}

To investigate the prognostic value of DEIRGs in SS patients, high-grade soft tissue sarcoma data in TCGA database were used to further screen the DEIRGs related to prognosis. Univariate Cox analysis was implemented by the survival package. Only these genes with a $\mathrm{P}$ value $<0.01$ were considered as prognostic immune-related genes.

\section{Construction of the immune-related prognostic signature for SS}

To develop a prognostic model, Lasso and multivariate Cox regression analyses were utilized to assess the relationship between DEIRGs expressions and overall survival (OS) or metastasis-free survival(MFS). risk_score $=\sum_{i=1}^{n}$ coe $f_{i} * X_{i}$. Coef refers to regression coefficient and $\mathrm{n}$ is the number of prognosticrelated DEIRGs.According to the formula, the risk score of each patient was calculated. Risk scores were acquired based on genes expression multiplied a linear combination of regression coefficient obtained from the multivariate Cox regression. Patients were assigned to high risk and low risk groups according to the median risk score. The Kaplan-Meier analysis was performed to compare overall survival between high risk and low risk groups via survival package in $\mathrm{R}$. Using the high grade soft tissue sarcoma dataset in TCGA, the receiver operating characteristic (ROC) curve was performed by the $\mathrm{R}$ software package survival ROC. In addition, univariate and multivariate analyses were utilized to assess the effect of risk scores on OS and MFS.

\section{Explorations of associations immune-related prognostic signature and immune cells infiltration.}

To explore the associations between prognostic model and immune cells infiltration, we performed single sample gene set enrichment analysis(ssGSEA).Twenty-nine immune marker gene sets were defined according to immune genome function (immune gene set included immune cell type, function, and pathway), and the enrichment level in each synovial sarcoma sample was quantified and ranked through sSGSEA. Furthermore, we conducted a Wilcoxon rank-sum test to compare the differential abundance of immune cells in the two groups which are according to the status of metastasis and risk score.

\section{Immune cell surface markers expression in SS patients}


To analyse the possible immune-related mechanisms of metastasis in patients with synovial sarcoma, we firstly analysed the correlation of immune checkpoint expression in the two groups of patients. We used GSE40021 data and clinical samples from our centre to analyse the expression of immune checkpoints in metastatic and nonmetastatic patients, including B and T-lymphocyte-associated protein (BTLA), programmed death receptor-1(PD-1),programmed death ligand-1(PD-L1), cytotoxic T-lymphocyteassociated protein 4(CTLA4), and lymphocyte-activation gene-3 (LAG-3).

The ssGSEA method indicated that CD4+ T lymphocytes and macrophages showed significantly high infiltration in patients with metastasis or patients with high-risk scores, while NK cells showed significantly low infiltration in these patients. Therefore, we used our clinical samples to perform RT-qPCR verification analysis of CD4, CD68, CD206, and CD56. The overall screening flow chart is shown in the flow chart.

\section{Data analysis}

All analyses were performed using $R$ (version 3.5.1). Unless otherwise noted, $P<0.05$ was considered significant.

\section{Results}

\section{Identification and characterization of DEIRGs in SS}

A total of 546 DEGs(225 upregulated and 321 downregulated) and 65 DEIRGs (8 upregulated and 57 downregulated) were identified as differentially expressed in metastatic SS compared with nonmetastatic patients. The heat maps revealed that metastatic SS can be obviously distinguished from the nonmetastatic patients according to DEGs and DEIRGs(Figure 1A, 1C). Volcano plots shows the distribution of differentially expressed genes between metastatic SS and nonmetastatic controls (Figure 1B, 1D).

The 65 DEIRGs were further analyzed by GO and KEGG analysis.GO analysis revealed that primary functional categories in the biological processes (BP) were T cell activation, $\mathrm{Y}$-interferon response, and antigen processing and presentation (Figure 2A). For cellular components (CC), the major enriched GO terms were $\mathrm{MHC}$ II protein complex, $\mathrm{MHC}$ protein complex, and clathrin-coated vesicle membrane (Figure 2B). The molecular functions (MF) mainly included the receptors ligands activity,peptide, amino and cytokine binding (Figure 2C).KEGG pathway indicated that the DEIRGs were mainly involved in human leukaemia virus- 1 infection, rheumatoid arthritis, antigen processing and presentation and other related pathways (Figure 2D).

Here, we utilized Cytoscape to construct and visualize the main regulatory network. As shown in Figure $2 \mathrm{E}$, protein-protein interactions(PPI) of DEGs were analysed using the STRING database.The most significant module in the PPI network was identified using the MCODE plugin. This protein regulatory network revealed the regulatory relationships among these immune-related genes. 


\section{Construction of the immune-related prognostic model for SS}

65 DEIRGs were subjected to Lasso Cox regression analysis, and 6 genes were filtered out. The results showed that only 5 upregulated genes (GREM2, CTSS, TINAGL1, ACKR1, and HLA-DRB1) and one downregulated gene (STC2) were independently related to prognosis. The OS and MFS were presented in Fig. 3A,3B.Then multivariate Cox analysis were performed and 6 genes were finally selected to establish a prognostic model. The formula was shown as: risk score $=(-0.056$ * GREM2 expression level $)+(-0.285$ * CTSS expression level $)+(0.297$ * STC2 expression level $)+(-0.180 *$ TINAGL1 expression level $)+$ $\left(0.003^{\star} \mathrm{ACKR} 1\right)+(0.052 \star \mathrm{HLA}$-DRB1 expression level).All the six genes were risky prognostic genes with hazard ratio $>1$. Risk scores were based on genes expression levels multiplied its corresponding regression coefficients. Regression coefficients were calculated by multivariate Cox regression. The risk scores were not only related to the expression levels of these genes, but also related to the correlation coefficients.

Then 263 high grade soft tissue sarcoma(STS) samples in TCGA were utilized again and randomly classified into a training set $(n=185)$ and validation set $(n=78)$. First, according to the formulas generated in the training set, the risk scores were calculated, including the risk score based on the OS signature. The ROC curves revealed that the discrimination of both signatures in the validation set and total set were favorable, with the AUC ranging from 0.694-0.721,0.702-0.729, respectively(Fig. 3C),indicating that the prognostic model had good sensitivity and specificity.Subsequently, according to the optimal risk score cutoff identified by X-tile, STS patients were stratified into high-risk and low-risk groups from training set and validation set. The K-M curves indicated that patients in the high-risk group had a worse OS than those in the low-risk group.These results revealed that both signatures were valuable tools for predicting the prognosis of STS patients.

\section{Immune-related prognostic model can predict immune cell infiltration}

Given the important roles of infiltrating immune cells in the tumour microenvironment, we integrated the comprehensive analysis of immune-related prognostic signature combined with immune infiltrates.

Based on the sSGSEA algorithm, we found that the infiltration of immune cells with differential abundance between the two groups which are according to the status of metastasis and risk score has a significant overlap(Figure 4A,4B).It is further indicating that the Immune-related prognostic model can assess the infiltration of immune cell well for SS.

Meanwhile,we explored the relationships between 6 hub immune signatures and several important immune cells.We observed that these signatures were associated with most of immune cells, which are also related to prognosis,especially activated CD4+T lymphocytes, macrophages, and NK cells(Figure 4C). 


\section{The metastatic mechanism of SS may not be related to the expression of immune checkpoints}

To further explore the relevant immune metastatic mechanism of SS, we utilized the GSE40021 dataset to divide patients into two groups (metastatic and nonmetastatic) and analysed the following immune checkpoints: BTLA4, CTLA4, PD-1, PD-L1, and LAG3. The results suggested that there was no significant difference in the expression of BTLA4, CTLA4 and PD-1. In addition, the expression of PD-L1 and LAG3 was lower in metastatic compared to nonmetastatic patients(Figure 5A). Previous clinical trials with subgroup analysis of SS have indicated that the PD-1 inhibitors has poor efficacy. Thus, we believe that the immune metastatic mechanism of SS is not caused by the surveillance pathways of evading immune checkpoints.

\section{Metastatic SS patient show significant expression of the GREM2 and CTSS genes, reduced activated NK cell and macrophage infiltration, and polarized M2 macrophages in the tumour microenvironment}

To further verify the results obtained through public database analysis, we collected 16 patients with SS who had follow-up data within one year in our centre (Clinical features are shown in Table 2). RNA was extracted from the tumour tissue, and the expression of DEIRGs (GREM2, CTSS, TINAGL1, ACKR1, HLADRB1, and STC2) was verified by real-time quantitative reverse transcription PCR(RT-qPCR). Compared to nonmetastatic patients, the GREM2 and CTSS genes were significantly high expression but the TINAGL1, ACKR1 and HLA-DRB1 was increased in metastatic SS. In addition, the expression of the STC2 gene was decreased in metastatic patients, but there was no significant difference between the two groups (Figure 5B).

To further explore the possible immune mechanism of metastatic SS, we performed RT-qPCR experiment to verify the expression of the immune cell surface marker.In preliminary work,we found the differences of immune cell infiltration between the two groups by the sSGSEA algorithm,and the results showed that there were nine types of immune cell were related to prognosis. We further selected which types of greatest difference in immune cell infiltration or survival prognosis for analysing. Finally, the following three types of cells were screened: activated CD4+ T lymphocytes, macrophages, and NK cells. We then selected their surface marker genes (CD4, CD68, CD206, and CD56) to verify the expression differences between the two groups by RNA levels. The RT-qPCR results indicated that the CD68 and CD56 genes were expressed at low levels in patients with metastatic SS, but the CD206 gene was highly expressed. These results were significantly different between the two groups. The expression of the CD4 gene tended to increase in patients with metastatic SS, but there was no significant difference (Figure 5C).

\section{Discussion}


Synovial sarcoma(SS) is a mesenchymal tumour manifested by different degrees of epithelial differentiation, including the formation of glands, presence of the specific ectopic $t(X ; 18)(p 11.2 ; q 11.2)$ gene, and production of the SS18-SSX fusion gene[13]. SS accounts for $5 \%-10 \%$ of soft tissue sarcomas.According to the recommendations of the NCCN and ASCO guidelines, the standardized treatment of early synovial sarcoma is mainly neoadjuvant chemotherapy combined with extensive surgical resection followed by chemotherapy or radiotherapy after surgery to strive for local radical treatment and reduce the occurrence of metastasis. However, even after standardized treatment, the 5year survival rate of patients is only $50 \%-60 \%$, and most of the patients die of lung metastasis, approximately $10 \%$ of patients have lung metastases at the first diagnosis. Even after extensive resection, approximately $50 \%$ of patients have lung metastasis during the follow-up process. Once lung metastasis occurs, the survival period is often less than 1 year, and the prognosis is extremely poor $[5,14]$. The highrisk time of recurrence or metastasis of synovial sarcoma peaks in the second year after surgery. After two years, the risk of local recurrence or metastasis is significantly reduced[15]. Several clinical trials have shown that several patients with high-grade sarcoma may benefit from immunotherapy. Thus, it is important to understand how to screen these patients and to identify relevant markers to predict the efficacy of immunotherapy[16]. At present, the metastatic mechanism of SS is still unclear, and there are no characteristic prognostic markers. Therefore, it is important to clarify the relevant molecular metastatic mechanisms of SS and to identify new immune-related therapeutic targets and prognostic markers.

In the study, we conducted a comprehensive analysis of the GSE40021 database to identify DEIRGs associated with metastatic SS, and we performed GO function and KEGG pathway analyses on DEIRGs. We then used TCGA database to analyse the survival of DEIRGs, and we constructed a risk assessment model to predict the metastasis of synovial sarcoma patients and patients who may benefit from immunotherapy, which has important reference value for clinical practice. GO function annotation showed that the DEIRGs mainly participated in the following functions: binding between cytokines and cytokine receptors,activation of $\mathrm{T}$ cells,antigen processing and presentation,activation of receptors and ligands, and participation of MHC class II protein complexes. KEGG pathway analysis showed that DEIRGs were mainly involved in human leukaemia virus-1 infection, rheumatoid arthritis, antigen processing and presentation, and other related pathways. These DEIRGs were closely related to the activation of the immune system, suggesting that the metastasis of SS may occur through immunerelated mechanisms. At the same time, tumour invasion and metastasis are extremely complex processes involving multiple steps and multiple genes. Therefore, the infiltration of different lymphocytes in the tumour microenvironment plays an important role in regulating tumour recurrence and metastasis. Zou et al. reported that Tregs have an immunosuppressive effect on cytotoxic CD8+ T lymphocytes through the adenosine A2A receptor pathway after activation of Tregs in the tumour microenvironment, ultimately leading to tumour recurrence or metastasis[17]. Schiavoni et al. found that the delay in lung metastasis of malignant melanoma may be caused by the increase in the infiltration of eosinophils and CD8+ T lymphocytes in the primary tumour through the IL-33/ST2 axis [18].Song et al. demonstrated that ovarian cancer cells highly express the ubiquitination-related protein(UBR5), and induce the recruitment and 
activation of tumour-associated macrophages by regulating the secretion of key chemokines and cytokines, leading to the failure of chemotherapy and immunotherapy, which is often associated with poor prognosis[19]. Therefore,the infiltration of immune cell that cause tumour metastasis varies in different tumour types.

At present, screening prognostic-related genes and constructing predictive models through multiomics sequencing have shown great predictive potential in a variety of tumour types. Li et al. constructed 14 immune-related gene prognostic risk assessment models, which showed good predictive performance for the prognosis of patients with osteosarcoma[20]. Huang et al. screened 15 alternative splicing genes related to prognosis and two splicing molecules related to bone metastasis to construct a risk assessment model, which showed that the prognosis of breast cancer patients and the occurrence of bone metastasis also have good predictive performance[21]. To the best of our knowledge, there is currently no risk assessment model for immune-related genes in patients with metastatic SS. The present study aimed to provide clinicians with accurate and important references for the prognosis of SS patients. The model contained six genes, namely, GREM2, CTSS, TINAGL1, ACKR1, HLA-DRB1 and STC2. GREM genes belong to the members of the DAN family, including Grem1 and Grem2, which are a large family that encode bone morphogenetic protein (BMP) antagonists. The secreted glycosylated protein encoded by this gene exerts an antagonistic effect by directly binding to the BMP protein, which plays an important regulatory role in organ formation and tissue differentiation[22, 23]. GREM2 is significantly highly expressed in gastric cancer tissues. Wang et al. demonstrated that it GREM2 maintains the stemness of gastric cancer cells through the JNK signalling pathway, ultimately promoting tumour invasion and metastasis[24]. Several studies have shown that the GREM2 gene also regulates the proliferation and differentiation of human pluripotent stem cell-derived cardiac progenitor cells by regulating the BMP signalling pathway[25,26], suggesting that this gene is closely related to the stemness of cells. The cathepsin S protein encoded by the CTSS gene is an important part of the family, and it is significantly different from other members of the cysteine protease family, mainly due to its limited tissue distribution and its ability to maintain good conformational stability at neutral and weakly alkaline $\mathrm{pH}$. Several studies have shown that CTSS is involved in the occurrence and development of tumours, such as tumour angiogenesis and metastasis[27, 28]. CTSS has also been found to be significantly highly expressed in tumour tissues or cell lines. At the same time, clinical evidence indicates that the upregulation of CTSS protein levels is related to the poor prognosis of tumour patients. Lee et al. found that the expression level of CTSS protein in the tumour tissues of breast cancer patients is significantly negatively correlated with the BRCA1 gene. These results suggest that the CTSS protein is activated after radiotherapy, which hydrolyses the BRCA1 protein, thereby inhibiting the repair of intracellular DNA double-strand break damage and eventually promoting tumour cell proliferation, invasion, and migration[29]. The STC2 gene encodes a secreted glycoprotein that regulates a variety of biological processes, such as the transport of calcium and phosphate in the kidney and intestine, cell metabolism, or cellular calcium/phosphate homeostasis. Several studies have shown that the STC2 gene regulates a variety of signalling pathways, and it is also closely related to cell proliferation, apoptosis, tumour metastasis, and treatment resistance. Wang et al. found that the STC2 gene is highly expressed in 
the tissues and metastatic lymph nodes of patients with nasopharyngeal carcinoma and that it is associated with poor prognosis[30]. At the same time, other studies have confirmed that the STC2 gene is closely related to the occurrence and development of hepatocellular carcinoma. In vivo experiments have confirmed that tumour cells activate the APAF1, APC, and PTEN pathways as well as reduce STC2 expression by knocking down the Mus81 gene, thereby inhibiting tumour cell proliferation and metastasis[31]. However, the above research results contradict our analysis. At present, the STC2 gene is mainly studied in epithelial-derived tumours. Considering that the role of STC2 in tumours of mesenchymal origin may be different from tumours of epithelial origin, subsequent biological experiments are still needed to confirm the inference.

To characterize the infiltration of immune cells in the tumour microenvironment, we further explored the relationship between immune-related prognostic models and immune cell infiltration. We found that activated B lymphocytes, effector memory CD4+ lymphocytes, effector memory CD8+ T lymphocytes, eosinophils, mast cells, monocytes, NK cells, plasma-like DC cells, and TH1 helper T lymphocytes had significantly low infiltration in patients with high-risk scores. Among them, eosinophils, monocytes, mast cells, and NK cells showed a significant difference in the metastasis-free survival time of low-risk patients, further suggesting that the immune-related prognostic model has a good ability to assess the infiltration of certain immune cells. At the same time, we found that there was a significantly low infiltration of macrophages and CD4+ T lymphocytes in patients with metastatic synovial sarcoma in the GSE40021 dataset. At present, there are few studies on mast cells and eosinophils in tumour metastasis. It remains unclear whether mast cells and eosinophils are involved in the regulation of the immune microenvironment of synovial sarcoma. Therefore, we focused on NK cells, macrophages, and CD4+ T lymphocytes.

Sutherland et al. found that the decrease in infiltration of NK cells rather than cytotoxic CD8+T lymphocytes in the tumour microenvironment is the main reason for the metastasis and spread of primary tumours by conducting a transcriptome analysis of the primary tumours of small cell lung cancer patients and transgenic mice[32]. Ren et al. found that the metastasis regulation of tumour-infiltrating neutrophils is mediated by the activation state of NK cells in a mouse model of breast cancer[33]. The infiltration of NK cells in tumours plays a crucial role in regulating tumour metastasis. Kuo et al. cocultured tumour cells with macrophages and collected the supernatant for proteomic analysis, and they showed that the tumour cells secrete succinate into the microenvironment and are activated by binding to succinate receptors on macrophages. Polarization to M2-type macrophages ultimately promotes tumour invasion and metastasis[34]. Ruffell et al. reported that macrophages are important participants in the regulation of tumour microenvironment homeostasis. Tumour cells promote the polarization of macrophages to the M2-type by secreting different mediators, which may be driven by local hypoxia and fibrosis of the tumour microenvironment. The increased infiltration of these M2-type macrophages leads to tumour recurrence and metastasis caused by inhibiting the recruitment of cytotoxic T lymphocytes and regulating other aspects of tumour immunity[35]. Sun et al. found that the high expression of the A2AR gene is closely related to the poor prognosis of patients, and high expression of the A2AR gene is also related to the high infiltration of CD4+ regulatory T lymphocytes (Tregs) by 
analysing the immunohistochemical results of large samples of head and neck squamous cell carcinoma tissues[36]. A recent review published by Lam et al. proposed that CD4+ T lymphocytes have a variety of different cell subpopulations. The balance between immunosuppressive Tregs and the proinflammatory TH17 cell subset plays an important regulatory role in the occurrence and metastasis of lung cancer. Several studies have shown that these two CD4+ $T$ cell subgroups play an active role in promoting lung cancer progression and metastasis[37]. The infiltration of NK cells, eosinophils, macrophages, and CD4+ $T$ lymphocytes in tumours plays a crucial role in regulating tumour metastasis. To further verify the role of these three immune cell subpopulations in patients with metastatic synovial sarcoma, we enrolled 16 synovial sarcoma patients with follow-up data in our centre from January 2018 to January 2019 in the study. Among them, 5 patients had metastases during the two-year follow-up period, and 11 patients had no metastases. In patients with metastatic SS, the infiltration of macrophages was significantly increased, and the infiltration of NK cells was significantly reduced. Furthermore, we also found that the infiltration of M2-type macrophages was the main infiltration, while the infiltration of CD4+ T lymphocytes was not significantly different. Combined with the analysis of the GEO database, we found that most of the immune checkpoints were not significantly different between metastatic and nonmetastatic SS patients. Moreover, there was low expression of LAG3 and PD-L1 in metastatic patients. These findings suggested that the metastasis of synovial sarcoma is not triggered by evading immune checkpoint surveillance. Combined with our analysis data, it is reasonable to speculate that the immune mechanism of metastasis may be caused by tumour cells inhibiting the secretion of certain cytokines, resulting in a decrease in the infiltration of NK cells and macrophages in the tumour microenvironment. We also found that the infiltrating macrophages were mainly polarized to the M2 type, which formed an immunosuppressive tumour microenvironment, thereby promoting lung metastasis.

Our study also had several limitations. First, the survival analyses of the identified DEIRGs were validated for all sarcomas and not specifically synovial sarcoma in TCGA because there is currently no database of large samples of synovial sarcoma patients with follow-up information. Peng et al. found that several biomarkers related to metastatic synovial sarcoma are also associated with prognosis through all sarcomas in TCGA. Because Peng and colleagues also found that the expression of the screening markers is significantly higher than that of normal tissues in the GEPIA dataset, they believe that this method of screening still has a certain reference value[38]. Existing evidence shows that immune-related prognostic models can predict SS metastasis and the infiltration of immune cell in the tumour microenvironment. However, the number of clinical samples was small, and future research will require a large number of clinical samples and multiomics analysis.

\section{Conclusion}

In conclusion,for the first time,several immunerelated genes were detected to be signifcantly related to SS prognosis by comprehensive analyses.Moreover,we constructed a novel immune-related prognostic model as an independent prognostic predictor for SS. This prognostic model may also serve as predictor for the immune cells infltration, proving its key role in tumor immune microenvironment. This may explain the poor efficacy of PD-1 inhibitors in synovial sarcoma. The mechanism of metastasis may be related to 
the polarization of macrophages towards the M2 type and remodelling of the immune microenvironment. Intervening in advance and reversing the changes in the immune microenvironment to increase the infiltration of NK cells is expected to delay metastasis and improve the survival of patients.

\section{Abbreviations}

SS: Synovial sarcoma; TCGA: The Cancer Genome Atlas; IRGs:Immune-related genes; DEGs: Differentially expressed genes; DEIRGs:Differentially expressed immune-related genes; RT-qPCR:Real-time quantitative reverse transcription; BTLA: B and T-lymphocyte-associated protein; PD-L1:programmed death receptor-1, PD-1:programmed death ligand-1; CTLA4:Cytotoxic T-lymphocyte-associated protein 4; LAG-

3:Lymphocyte-activation gene-3; GO: Gene ontology database; CC:cellular component;BP:biological process;MF:molecular function;KEGG: The Kyoto encyclopedia of genes and genomes; ROC: Receiver operating characteristic; AUC: Area under the ROC curve; OS: Overall survival. MFS:Metastasis-free survival analysis;LMS:Leiomyosarcoma; DLPS:Dedifferentiated;liposarcoma; UPS:undifferentiated pleomorphic sarcoma; MFS:Myxofibrosarcoma; MPNST:Malignant Peripheral Nerve Sheath Tumor

\section{Declarations}

\section{Acknowledgements}

The authors would like to express our sincere thanks for sharing the data from The Cancer Genome Atlas (TCGA) ,GEO and ImmPort gene database.

\section{Authors' contributions}

HYF and LJC conceived the study and performed the bioinformatics analyses. HYF and GM downloaded and organized the clinical and gene expression data. GM performed the statistical analyses. HYF and GM wrote the manuscript. WJ ,TQL and ZXJ critically revised the article for essential intellectual content and administrative support. All authors read and approved the final manuscript.

\section{Funding}

Grant agency: Cultivating fund for Chinese National Natural Science Foundation of Sun Yat-sen University Cancer Center.

Grant Number: This study was supported by the National Natural Science Foundation of China (91959115, 81872268,and 81702665), Natural Science Foundation of Guangdong Province (2019A1515011192) and the Fundamental Research Funds for the Central Universities (19ykpy189). Each of the funding bodies granted the funds on the basis of a research proposal. The bodies had no influence on the experimental design, data analysis and interpretation, or writing of the manuscript.

\section{Availability of data and materials}


Not applicable.

\section{Ethics approval and consent to participate}

The studies involving human participants were reviewed and approved by the Medical Ethics Committee of the Sun Yat-sen University Cancer Center. Written informed consent to participate in this study was provided by the participants' legal guardian/next of kin.

\section{Consent for publication}

Not applicable.

\section{Competing interests}

The authors declare that they have no competing interests.

\section{References}

1. Choi JH, Ro JY. The 2020 WHO Classification of Tumors of Soft Tissue: Selected Changes and New Entities. Adv Anat Pathol. 2021;28(1):44-58.

2. Ferrari A, Gronchi A, Casanova M, Meazza C, Gandola L, Collini P et al. Synovial sarcoma: a retrospective analysis of 271 patients of all ages treated at a single institution. Cancer. 2004;101(3):627-34.

3. Singer S, Baldini EH, Demetri GD, Fletcher JA, Corson JM. Synovial sarcoma: prognostic significance of tumor size, margin of resection, and mitotic activity for survival. J Clin Oncol. 1996;14(4):1201-8.

4. Aparna M, Natarajan J, Arumugam C, Radhakrishnan R. Primary synovial sarcoma of the maxilla. J Cancer Res Ther. 2014;10(3):739-41.

5. Eilber FC, Dry SM. Diagnosis and management of synovial sarcoma. J Surg Oncol. 2008;97(4):31420.

6. Tawbi HA, Burgess M, Bolejack V, Van Tine BA, Schuetze SM, Hu J et al. Pembrolizumab in advanced soft-tissue sarcoma and bone sarcoma (SARC028): a multicentre, two-cohort, single-arm, open-label, phase 2 trial. Lancet Oncol. 2017;18(11):1493-501.

7. D'Angelo SP, Mahoney MR, Van Tine BA, Atkins J, Milhem MM, Jahagirdar BN et al. Nivolumab with or without ipilimumab treatment for metastatic sarcoma (Alliance A091401): two open-label, noncomparative, randomised, phase 2 trials. Lancet Oncol. 2018;19(3):416-26.

8. D'Angelo SP, Melchiori L, Merchant MS, Bernstein D, Glod J, Kaplan R et al. Antitumor Activity Associated with Prolonged Persistence of Adoptively Transferred NY-ESO-1 (c259)T Cells in Synovial Sarcoma. Cancer Discov. 2018;8(8):944-57.

9. Bhattacharya S, Andorf S, Gomes L, Dunn P, Schaefer H, Pontius J et al. ImmPort: disseminating data to the public for the future of immunology. Immunol Res. 2014;58(2-3):234-9.

10. The Gene Ontology (GO) project in 2006. Nucleic Acids Res. 2006;34(Database issue):D322-6. 
11. Kanehisa M, Furumichi M, Tanabe M, Sato Y, Morishima K. KEGG: new perspectives on genomes, pathways, diseases and drugs. Nucleic Acids Res. 2017;45(D1):D353-d61.

12. Shannon P, Markiel A, Ozier O, Baliga NS, Wang JT, Ramage D et al. Cytoscape: a software environment for integrated models of biomolecular interaction networks. Genome Res. 2003;13(11):2498-504.

13. Mitchell G, Pollack SM, Wagner MJ. Targeting cancer testis antigens in synovial sarcoma. J Immunother Cancer. 2021;9(6).

14. DeSalvo J, Ban Y, Li L, Sun X, Jiang Z, Kerr DA et al. ETV4 and ETV5 drive synovial sarcoma through cell cycle and DUX4 embryonic pathway control. J Clin Invest. 2021;131(13).

15. Lagarde P, Przybyl J, Brulard C, Pérot G, Pierron G, Delattre $O$ et al. Chromosome instability accounts for reverse metastatic outcomes of pediatric and adult synovial sarcomas. J Clin Oncol. 2013;31(5):608-15.

16. Stacchiotti S, Van Tine BA. Synovial Sarcoma: Current Concepts and Future Perspectives. J Clin Oncol. 2018;36(2):180-7.

17. Maj T, Wang W, Crespo J, Zhang H, Wang W, Wei S et al. Oxidative stress controls regulatory T cell apoptosis and suppressor activity and PD-L1-blockade resistance in tumor. Nat Immunol. 2017;18(12):1332-41.

18. Lucarini V, Ziccheddu G, Macchia I, La Sorsa V, Peschiaroli F, Buccione C et al. IL-33 restricts tumor growth and inhibits pulmonary metastasis in melanoma-bearing mice through eosinophils. Oncoimmunology. 2017;6(6):e1317420.

19. Song M, Yeku OO, Rafiq S, Purdon T, Dong X, Zhu L et al. Tumor derived UBR5 promotes ovarian cancer growth and metastasis through inducing immunosuppressive macrophages. Nat Commun. 2020;11(1):6298.

20. Li LQ, Zhang LH, Zhang Y, Lu XC, Zhang Y, Liu YK et al. Construction of immune-related gene pairs signature to predict the overall survival of osteosarcoma patients. Aging (Albany NY). 2020;12(22):22906-26.

21. Huang R, Guo J, Yan P, Zhai S, Hu P, Zhu X et al. The Construction of Bone Metastasis-Specific Prognostic Model and Co-expressed Network of Alternative Splicing in Breast Cancer. Front Cell Dev Biol. 2020;8:790.

22. Joyce JA, Hanahan D. Multiple roles for cysteine cathepsins in cancer. Cell Cycle. 2004;3(12):1516619.

23. Jedeszko C, Sloane BF. Cysteine cathepsins in human cancer. Biol Chem. 2004;385(11):1017-27.

24. Ran A, Guan L, Wang J, Wang Y. GREM2 maintains stem cell-like phenotypes in gastric cancer cells by regulating the JNK signaling pathway. Cell Cycle. 2019;18(19):2414-31.

25. Tanwar V, Bylund JB, Hu J, Yan J, Walthall JM, Mukherjee A et al. Gremlin 2 promotes differentiation of embryonic stem cells to atrial fate by activation of the JNK signaling pathway. Stem Cells. 2014;32(7):1774-88. 
26. Bylund JB, Trinh LT, Awgulewitsch CP, Paik DT, Jetter C, Jha R et al. Coordinated Proliferation and Differentiation of Human-Induced Pluripotent Stem Cell-Derived Cardiac Progenitor Cells Depend on Bone Morphogenetic Protein Signaling Regulation by GREMLIN 2. Stem Cells Dev. 2017;26(9):67893.

27. Flannery T, Gibson D, Mirakhur M, McQuaid S, Greenan C, Trimble A et al. The clinical significance of cathepsin S expression in human astrocytomas. Am J Pathol. 2003;163(1):175-82.

28. Kos J, Sekirnik A, Kopitar G, Cimerman N, Kayser K, Stremmer A et al. Cathepsin S in tumours, regional lymph nodes and sera of patients with lung cancer: relation to prognosis. $\mathrm{Br} \mathrm{J}$ Cancer. 2001;85(8):1193-200.

29. Kim S, Jin H, Seo HR, Lee HJ, Lee YS. Regulating BRCA1 protein stability by cathepsin S-mediated ubiquitin degradation. Cell Death Differ. 2019;26(5):812-25.

30. Li J, Zhang Z, Feng X, Shen Z, Sun J, Zhang X et al. Stanniocalcin-2 promotes cell EMT and glycolysis via activating ITGB2/FAK/SOX6 signaling pathway in nasopharyngeal carcinoma. Cell Biol Toxicol. 2021.

31. Wu F, Li TY, Su SC, Yu JS, Zhang HL, Tan GQ et al. STC2 as a novel mediator for Mus81-dependent proliferation and survival in hepatocellular carcinoma. Cancer Lett. 2017;388:177-86.

32. Best SA, Hess JB, Souza-Fonseca-Guimaraes F, Cursons J, Kersbergen A, Dong X et al. Harnessing Natural Killer Immunity in Metastatic SCLC. J Thorac Oncol. 2020;15(9):1507-21.

33. Li P, Lu M, Shi J, Hua L, Gong Z, Li Q et al. Dual roles of neutrophils in metastatic colonization are governed by the host NK cell status. Nat Commun. 2020;11(1):4387.

34. Wu JY, Huang TW, Hsieh YT, Wang YF, Yen CC, Lee GL et al. Cancer-Derived Succinate Promotes Macrophage Polarization and Cancer Metastasis via Succinate Receptor. Mol Cell. 2020;77(2):21327.e5.

35. DeNardo DG, Ruffell B. Macrophages as regulators of tumour immunity and immunotherapy. Nat Rev Immunol. 2019;19(6):369-82.

36. Ma SR, Deng WW, Liu JF, Mao L, Yu GT, Bu LL et al. Blockade of adenosine A2A receptor enhances CD8(+) T cells response and decreases regulatory $T$ cells in head and neck squamous cell carcinoma. Mol Cancer. 2017;16(1):99.

37. Marshall EA, Ng KW, Kung SH, Conway EM, Martinez VD, Halvorsen EC et al. Emerging roles of T helper 17 and regulatory $T$ cells in lung cancer progression and metastasis. Mol Cancer. 2016;15(1):67.

38. Song Y, Liu X, Wang F, Wang X, Cheng G, Peng C. Identification of Metastasis-Associated Biomarkers in Synovial Sarcoma Using Bioinformatics Analysis. Front Genet. 2020;11:530892.

\section{Tables}

Due to technical limitations, tables are only available as a download in the Supplemental Files section. 
A

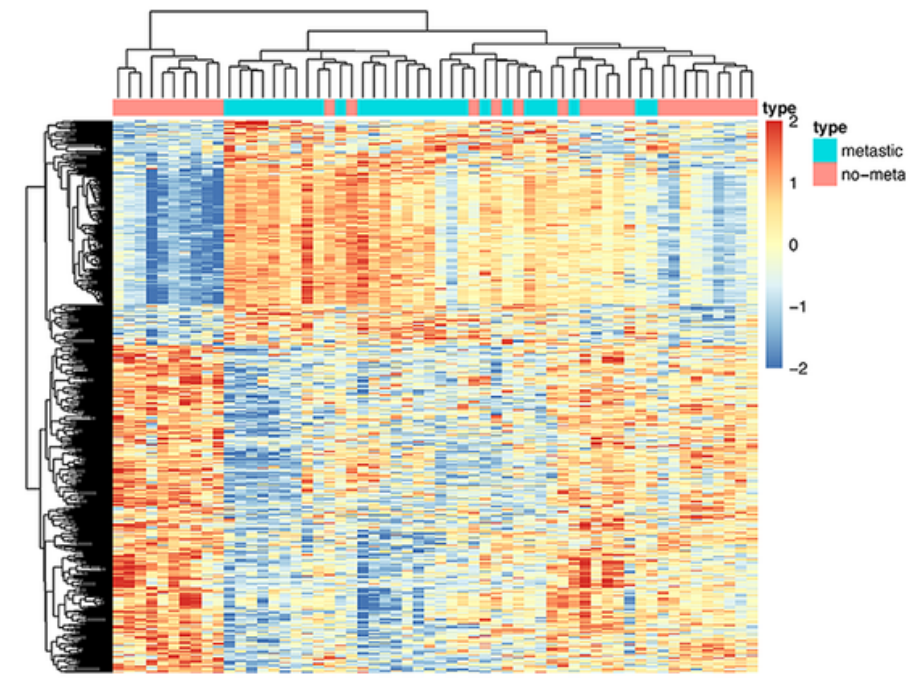

C

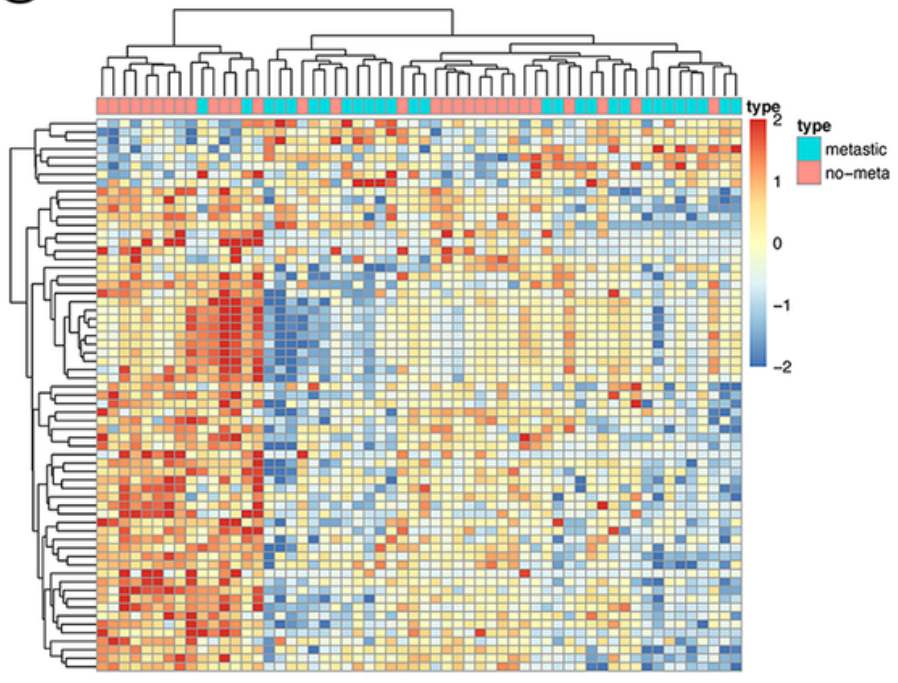

B

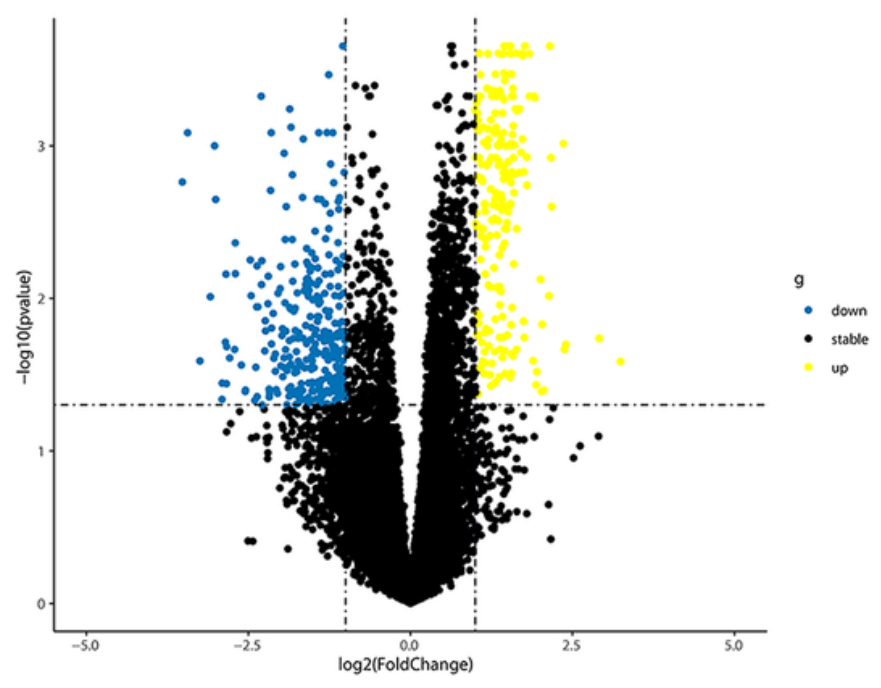

D

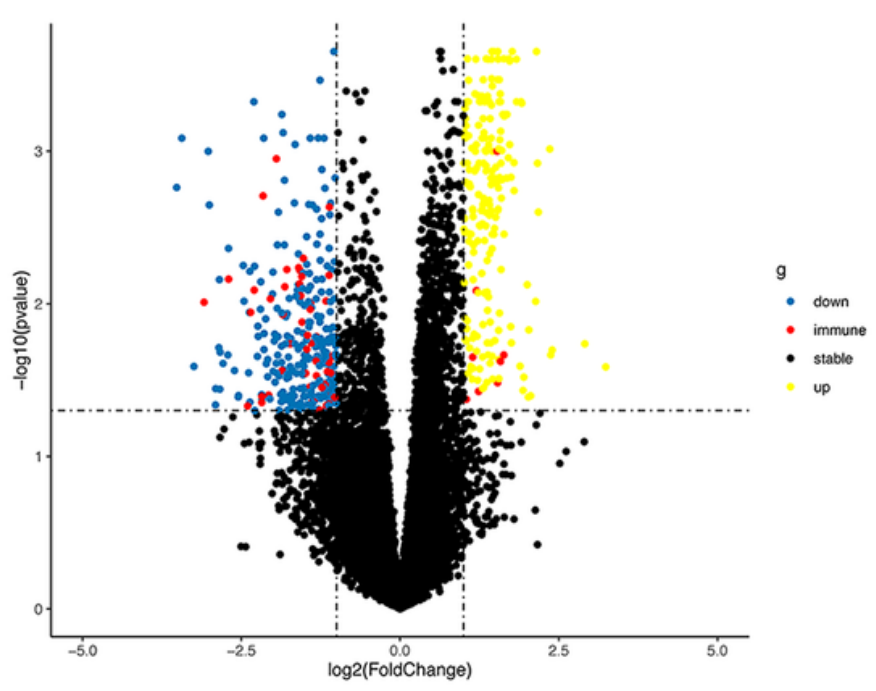

Figure 1

Identification of differentially expressed immune-related genes (DEIRGs) associated with SS metastasis. Heatmap of the top (A) 546 DEGs and (C) 65 DEIRGs associated with SS metastasis in the GE040021 datasets. The colour from blue to red represents the progression from low expression to high expression. Volcano plot of (B) DEGs and (D) DEIRGs detected from the GE040021 datasets. The yellow dots represent upregulated genes with statistical significance, and the blue dots represent downregulated genes with statistical significance. The red dots represent immune-related genes with statistical significance, and the black dots represent no differentially expressed genes. 

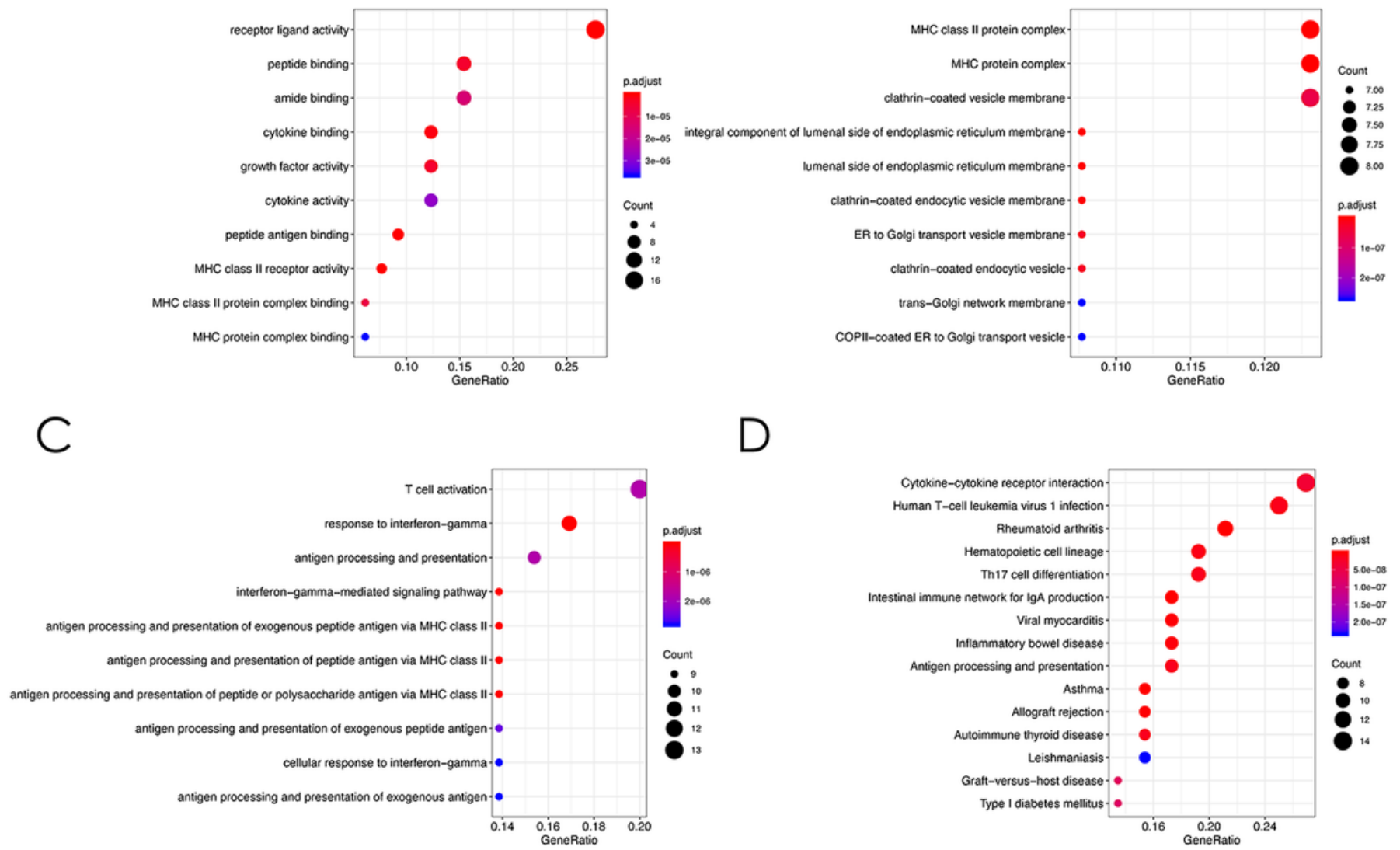

$\mathrm{E}$
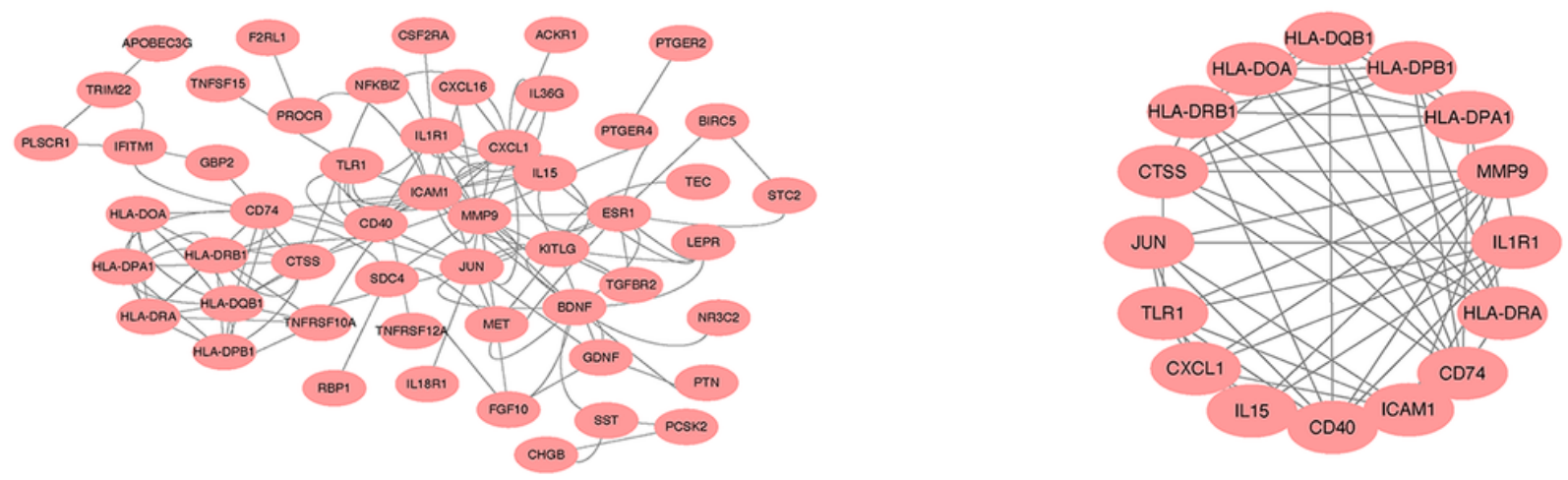

\section{Figure 2}

Functional enrichment analysis of differentially expressed immune-related genes(DEIRGs). Gene Ontology analysis representing (A) biological process, (B) cellular component, and (C) molecular function. (D) The top 10 most significant Kyoto Encyclopaedia of Genes and Genomes (KEGG) pathways. (E) Protein-protein interactions of DEGs were analysed using the STRING database. The most significant module in the PPI network was identified using the MCODE plugin. 
A

GREM2 + $\mathrm{Kgh}+\mathrm{Low}$

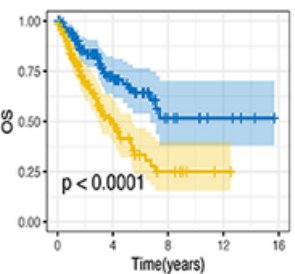

CTSS $+\mathrm{Hghn}+$ Lom

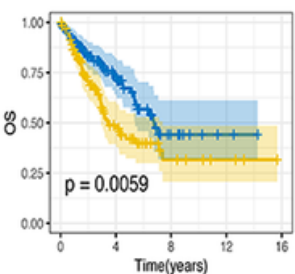

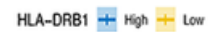

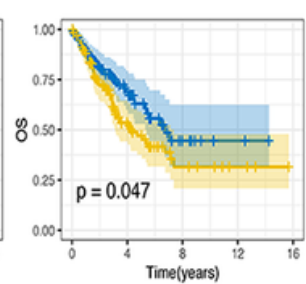

C

Training set $(n=185)$

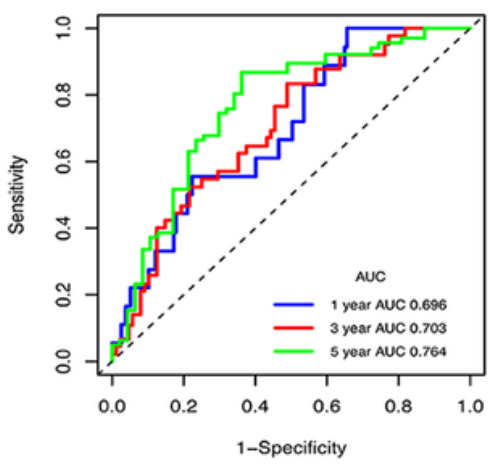

survival curve $(p=0.0000122241)$

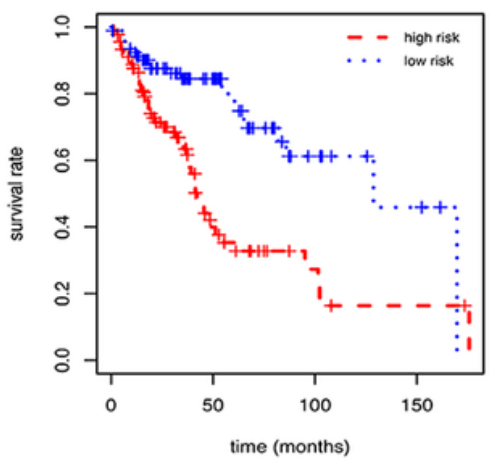

B

ACKR1 + $\mathrm{High}_{+}+\mathrm{Low}$

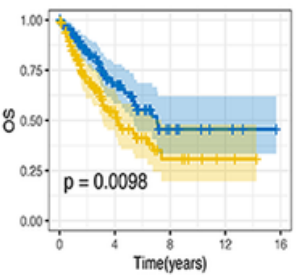

TINAGL1 + High + Low

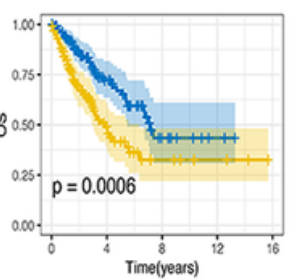

GREM2 + High + Low

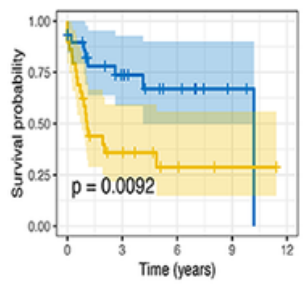

CTSS + High + Low

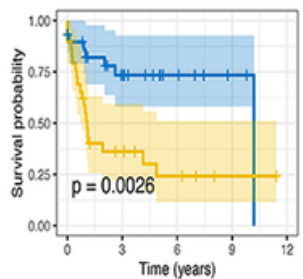

$\mathrm{STC2}+\mathrm{Hgh}+$ Low

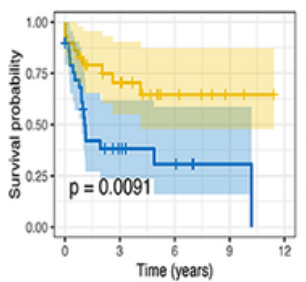

HLA-DRB1 $+\mathrm{Hgh}+$ Low

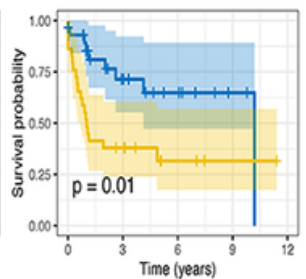

ACKR1 + $\mathrm{Hgh}+$ Low

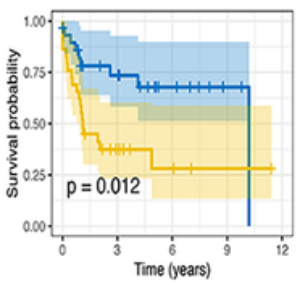

TINAGL1 $+\mathrm{High}+$ Low

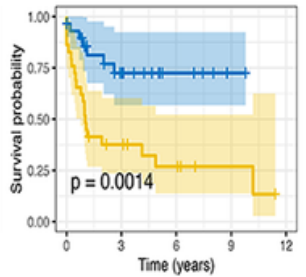

Total set $(n=263)$
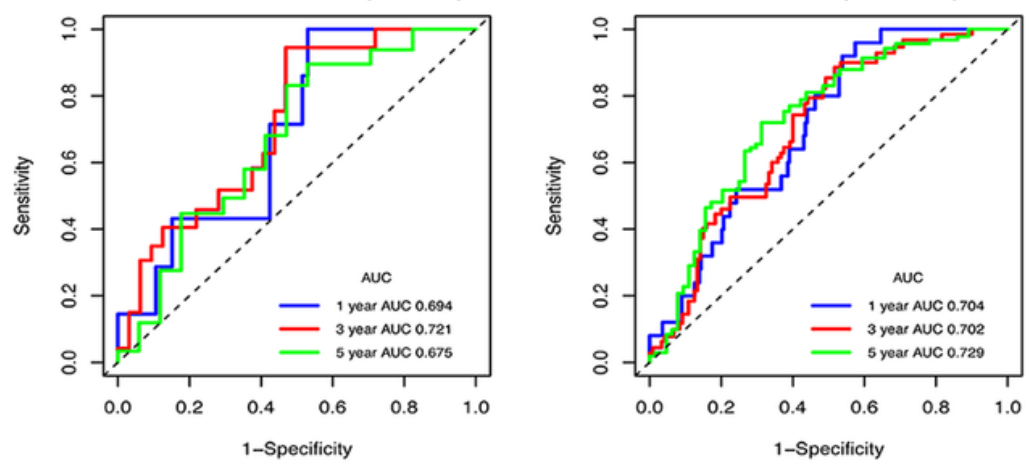

survival curve $(p=0.0362683426)$
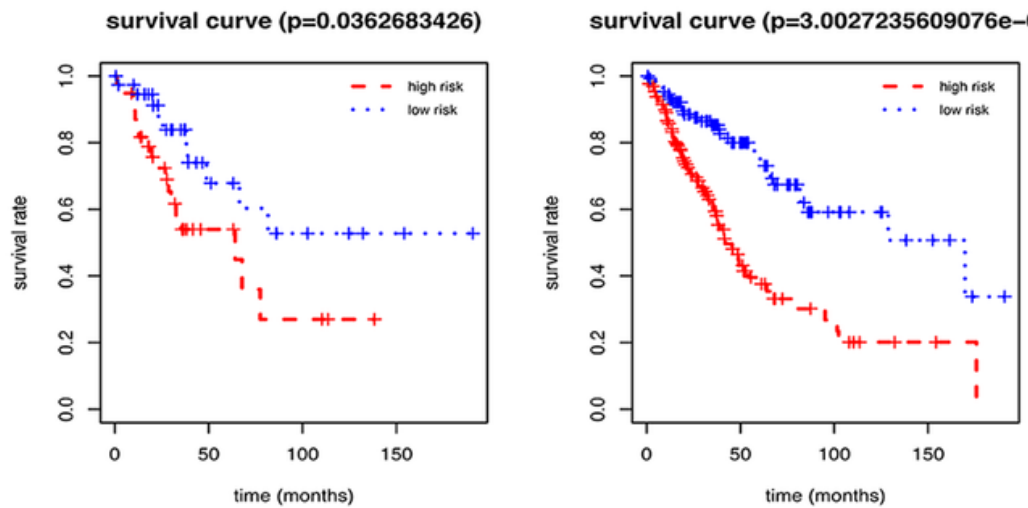

\section{Figure 3}

Construction of an immune-related prognostic model for synovial sarcoma. Kaplan-Meier curves for (A) overall survival and (B) metastasis-free survival analysis of the DEIRGs in SS patients. The blue line represents the high expression group, and the yellow line denotes the low expression group. The figure shows the Kaplan-Meier curves of the six genes screened with significant differences. (C) A total of 185 patients were included in the training set, and the remaining 78 patients were included in the validation set. Receiver operating characteristic curve (ROC) analysis predicted overall survival using the risk score. Time-dependent ROC curves of the OS signatures at 1,3, and 5 years. Survival curves for the low-risk and 
high-risk groups. The red line represents the high-risk score group, and the blue line denotes the low-risk score group.

A

B

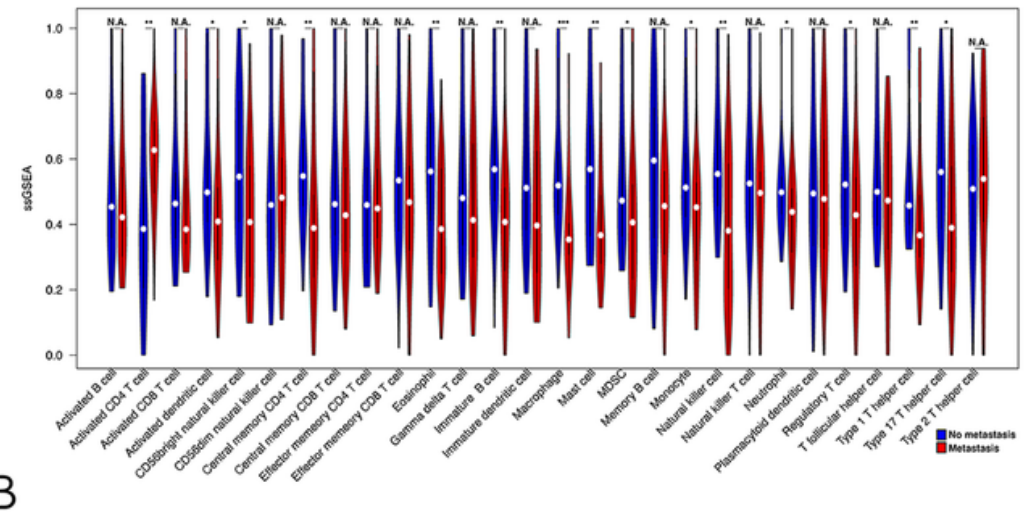

C
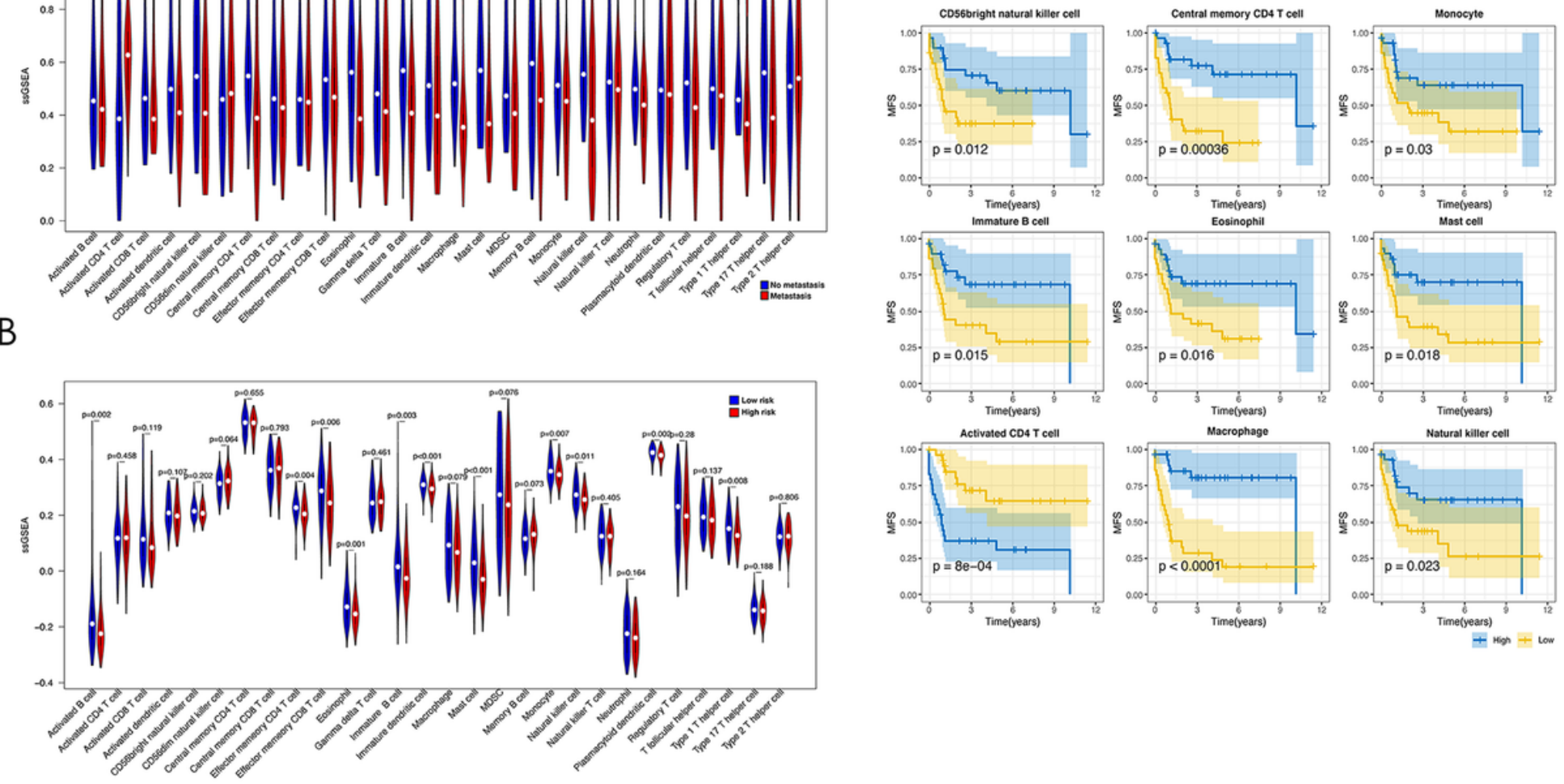

Figure 4

Analysis of the immune landscape of GE040021 patients. Immune infiltration landscape analysed by the ssGSEA score-based method in synovial sarcoma from the GE040021 dataset. (A) The blue square represents the no metastasis group, and the red square denotes the metastasis group. (B) The blue square represents the high-risk-score group, and the red square denotes the low-risk-score group. (C) Infiltrating immune cells were significantly associated with improved prognosis. The high- and low-score groups were divided based on the top $30 \%$ and the bottom $30 \%$ infiltrating scores calculated by the ssGSEA algorithm, respectively. 
A

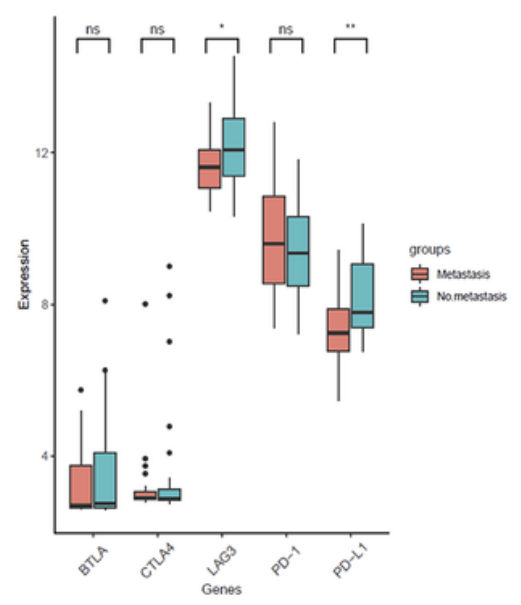

C
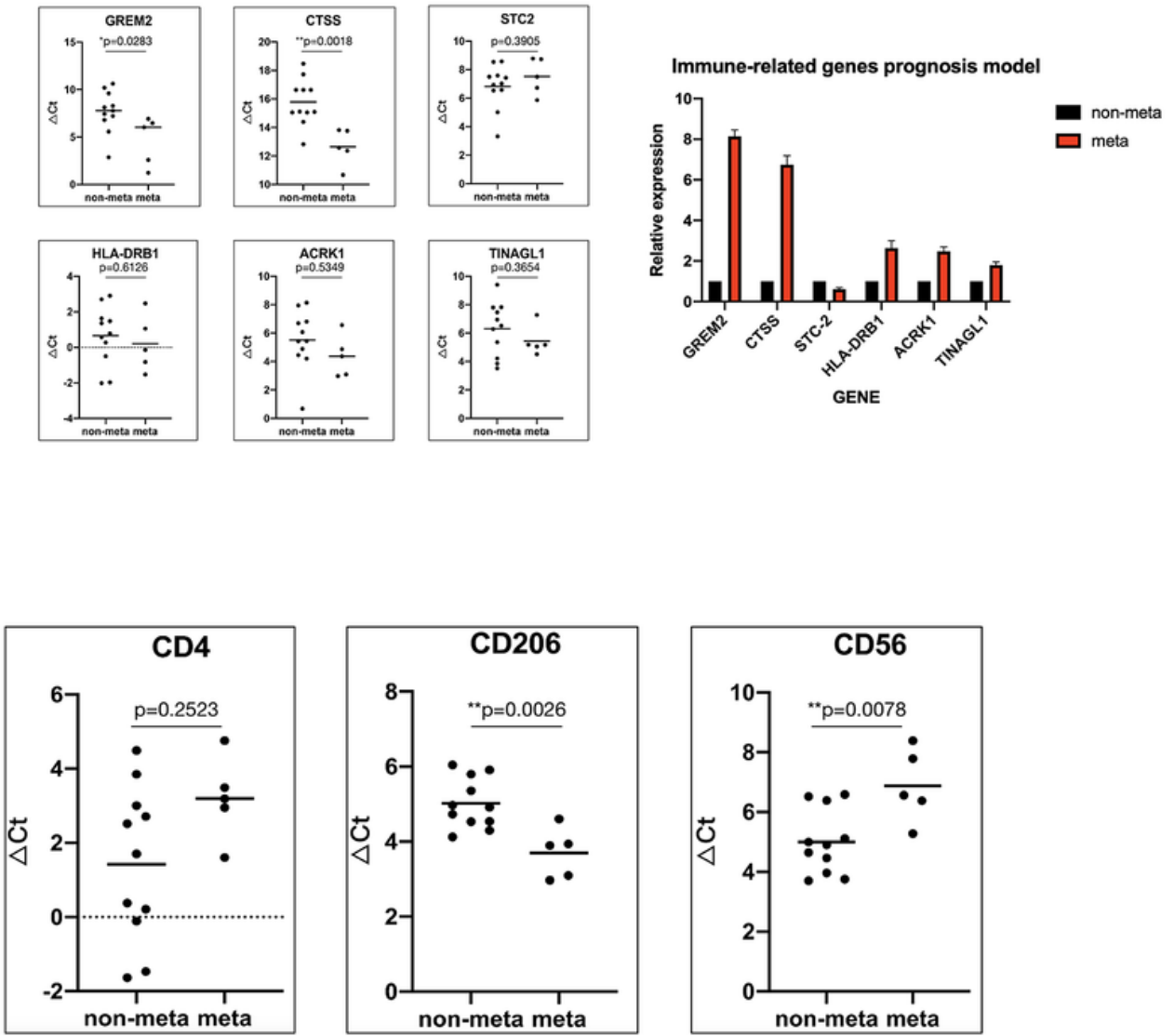

\section{Figure 5}

The immune mechanism of metastatic synovial sarcoma may be due to the reduction of NK cell and macrophage infiltration in the tumour microenvironment as well as polarization of macrophages towards $M 2$, forming an immunosuppressive microenvironment. (A) Comparison showing the mRNA expression levels of immune checkpoints in the GE040021 dataset. (B) Sixteen synovial sarcoma patient samples were used to verify the mRNA expression levels of six genes, which were used to construct an immunerelated risk assessment model. (C) mRNA expression levels of immune cell markers. CD68, total macrophage surface marker; CD4, total helper T cell surface marker; CD206, M2-type macrophage surface marker; and CD56, total NK cell surface marker.

\section{Supplementary Files}

This is a list of supplementary files associated with this preprint. Click to download.

- Table1.png

- Table2.png

- Flowchartofstudy.png 